Vol. 7(4), pp. 48-53, April 2015

DOI: $10.5897 /$ J DOH2015.0141

Article Number: D1DE2BF51775

ISSN: 2141-2472

Copyright (C) 2015

Author(s) retain the copyright of this a ricle

http://www.academic joumals.org/J DOH

Journal of Dentistry and Oral Hygiene

Full Length Research Paper

\title{
Is there hidden caries or is this a limitation of the conventional exams?
}

\author{
Valdeci Elias dos Santos Junior*, Arnoldo Vasconcelos Filho, Andreza Cristina de Lima \\ Targino Massoni and Aronita Rosenblatt
}

Department of Paediatric Dentistry, Faculty of Dentistry, University of Pernambuco, Brazil.

Received 23 February, 2015; Accepted 23 March, 2015

\begin{abstract}
The aim of this study was to verify the existence of hidden caries and compare the diagnosis agreement among different examiners by visual, dental radiography and computed microtomography images of hidden dental caries lesions on the occlusal surface of extracted molars. Two hundred and fifteen teeth were examined visually and extracted from cadavers of adolescents with a mean age of 12 years. Eleven teeth which showed hidden occlusal caries were included in the study. Occlusal sites were examined both visually and by dental radiograph by 3 different dental practitioners. Results were compared and validated independently by each examiner with computed microtomography; hence, the gold-standard in current investigation. The coefficient of agreement was calculated by the Cohen's Kappa test. The agreement among examiners for the diagnosis by standard microtomography was excellent $(K=0.924)$ and moderate for both the visual $(K=0.515)$ and the radiographic $(K=0.583)$ examinations. Computed microtomography-produced images which allowed the examiners to visualize radiolucent areas unseen in previous radiographic images and visual examinations showed anatomic communication between enamel and/or dentine to the external environment, not necessarily due to enamel collapse. Occlusal hidden caries was being over detected by clinicians because of the limitations of the conventional dental visual and radiographic examination, which indicate that the examinations are not accurate enough to detect the lesions.
\end{abstract}

Key words: Computed microtomography, dental diagnostics, dental caries.

\section{INTRODUCTION}

Since 1970, the pattern and prevalence of dental caries in permanent teeth have revealed a marked change (McDonald and Sheiham, 1992; Beltrán-Aguilar et al., 1999; Whelton, 2004; Bernabé, 2009; Bagramian et al., 2009). This fact indicates that dentine lesions under noncavitated enamel actually represent about $50 \%$ of total caries in many world communities (Bagramian et al., 2009). Although several labels for this type of lesion have been forwarded, namely, 'fluoride syndrome', 'fluoride bomb' and 'hidden caries' (Weerheijm, 1997; Zadik and Bechor, 2008; Hashizume et al., 2013), its aetiology is still undetermined.

*Corresponding author. E-mail: va ldeciodonto@gmail.com.

Author(s) agree that this artic le remain pemanently open access under the terms of the $\underline{C}$ reative Commons Attribution License 4.0 Intemational License 
Dentists who routinely examine clinically caries-free children may be shocked to discover a large caries lesion on a radiograph that they may have missed in the visual examination (Bader et al., 2002; Mutsvari et al., 2012). The detection rate of such lesions depends upon the prevalence of caries in the population and on the frequency of bite-wing radiographic examinations. Information on the prevalence of these lesions in the world population is still unpractised, even though the available data shows that it ranges from $0.8 \%$ in Scotland (Creanor et al., 1990) to $50 \%$ in Germany (Weerheijm et al., 1992).

The visual examination is the method of choice to diagnose dental caries. However, clinically undetected occlusal caries are of difficult diagnosis (Bader et al., 2002) and routinely require additional examinations, such as conventional radiographs (Souza-Zaroni et al., 2006; Ricketts et al., 2007) for a more accurate one. However, the aforementioned technique's limitation results from overlapping images of the occlusal surface due to a twodimension diagnosis (Neuhaus et al., 2009).

While the x-ray method projects the image on two planes, the CT-scan image reproduces the internal structures on three spatial planes. Thus, the structural relations are shown in depth, including the images of the segments and layers' internal structures, particularly the mineralized tissues, with excellent definition, which favor the division of three dimensional irregularities (Parks, 2000).

Thus, the aim of this study was to verify the existence of hidden caries and compare the diagnosis agreement among different examiners between the visual and radiographic examination and computed microtomography.

\section{METHODOLOGY}

In this in vitro descriptive study, 215 permanent molars were collected from donated cadavers of 12 to 15 -year-old adolescents at the Forensic Medicine Institute of Pernambuco, Brazil and 11 $(5.11 \%)$ of the 215 teeth were cleaned, dried and included in the study, the ones that exhibited caries images under microtomography examination (micro-CT scan).

The investigation was developed according to recommendations by the Committee in Ethics in Human Research of the University of Pernambuco, Brazil. Furthermore, parents or legal representatives agreed on the donation of the teeth and signed the informed consent.

\section{Detection of caries without communication with the external environment}

Due to their three-dimensional nature, CT scanning images were used as standard to detect the existence of lesions in dentine without any external communication. The teeth were then examined by three independent examiners who looked at the images and identified the absence (0) or presence (1) of the lesions. Examiner 1 was the gold standard on detecting caries in micro-CT scan and participated in all examinations.

\section{Accuracy of diagnosis tests}

Specimens were prepared by carefully cleaning the teeth with hand scale and rotating bristle brushes with water/pumice to remove deposits of calculus, plaque or debris. They were then stored in individually identified bottles with $10.0 \%$ formaldehyde solution.

The teeth were examined by three independent examiners employing visual radiographic and microtomographic methods. The three examiners were identified as Examiner 1, Examiner 2 and Examiner 3.

\section{Visual examination (VE)}

Visual examination was performed using a dental light unit. Three independent dentists, averaging 10 years clinical experience each, examined each tooth, looked at the occlusal surfaces and identified the absence (0) or presence (1) of cavitated caries lesions.

\section{Radiographic examination (RE)}

For the radiographic examination, the teeth were held with utility wax and radiographed with periapical film (Ektaspeed/Eastman Kodak Company ${ }^{\circledR}$, Rochester, USA). The focus-film distance was $20 \mathrm{~cm}$, with an exposure time of $0.5 \mathrm{~s}$. Radiographs were taken using Gnatus X-ray equipment (10 mA and $70 \mathrm{kV})$ and the films were processed in a portable dark-box and in solutions (developer and fixer) for conventional Kodak Dental X-rays (Kodak ${ }^{\circledR}$, São José dos Campos SP Brazil), at a temperature of $24^{\circ} \mathrm{C}$, for $3 \mathrm{~min}$. Defective radiographs were discarded and new radiographs taken. The teeth were then examined by three independent examiners who looked at the images and identified the absence (0) or presence (1) of the dentine lesions.

\section{Computed microtomographic examination (MCTSE)}

Teeth were imaged by micro-CT scan on a Siemens Inveon PETCT (Siemens Molecular Imaging, Munich, Germany) for microtomographic examination. The x-ray source was set to voltage and current of $80 \mathrm{kVp}$ and $500 \mu \mathrm{A}$, respectively. The x-ray source and $C C D$ detector camera were positioned to each tooth so that the effective pixel size would be $41.80 \mu \mathrm{m}$ isotropically. As many as 360 projections were retrieved for a 330-min exposure time. All projection data were reconstructed with Filtered Backprojection. The images were interpolated bilinearly, and filtered with a SheppLogan filter for higher resolution. Finally, conversion to DICOM format allowed the images to be viewed by DICOM medical imaging viewers.

The teeth were then examined by three independent examiners who looked at the images and identified the absence (0) or presence (1) of enamel and dentine lesions.

\section{Statistical analysis}

Because of its superior image quality over the other two methods, the Micro-CT was chosen as the gold-standard method to compare the accuracy of the micro-CT scan with the visual and radiographic examination of the occlusal lesions.

Moreover, the inter-examiners agreement for the tested methods (visual, radiographic and micro-CT scan) for occlusal lesion detection was also calculated and thus Cohen's Kappa statistic test expressed the coefficient of agreement for the three examiners.

The Kappa statistic test, formulated by Cohen in 1960, is an agreement coefficient that corrects the error due to chance by paired analysis. It compares the observed proportion of agreement 
Table 1. Cross-tables showing the relationship between the different methods and Cohen's kappa values for Inter-observer for occlusal lesion. detection.

\begin{tabular}{|c|c|c|c|c|c|}
\hline \multirow{2}{*}{ Variable } & \multicolumn{3}{|c|}{ Examiners } & \multirow{2}{*}{ Total } & \multirow{2}{*}{ kappa IE } \\
\hline & 1 & 2 & 3 & & \\
\hline \multicolumn{6}{|l|}{ Visual } \\
\hline (0) Absence & 7 & 6 & 6 & 19 & \\
\hline (1) Presence & 4 & 5 & 5 & 14 & 0.515 \\
\hline Total & 11 & 11 & 11 & 33 & \\
\hline \multicolumn{6}{|l|}{ Radiography } \\
\hline (0) Absence & 3 & 3 & 4 & 10 & \\
\hline (1) Presence & 8 & 8 & 7 & 23 & 0.583 \\
\hline Total & 11 & 11 & 11 & 33 & \\
\hline \multicolumn{6}{|c|}{ Micro-CT scan } \\
\hline (0) Absence & 1 & 2 & 1 & 3 & \\
\hline (1) Presence & 10 & 9 & 10 & 6 & 0.924 \\
\hline Total & 11 & 11 & 11 & 33 & \\
\hline
\end{tabular}

Table 2. Coincidence between visual examination and radiographic examination in relation to micro-CT scan examination for three Examiners.

\begin{tabular}{lccc}
\hline \multirow{2}{*}{ Variable } & \multicolumn{2}{c}{ Concordance } & Total \\
\cline { 2 - 3 } & Yes & No & \\
\hline Visual $\times$ Micro-CT scan & 3 & 8 & 11 \\
Examiner A & 6 & 5 & 11 \\
Examiner B & 6 & 5 & 11 \\
Examiner C & 16 & 17 & 33 \\
Total & & & \\
Radiography $\times$ Micro-CT scan & 2 & 9 & 11 \\
Examiner A & 3 & 8 & 11 \\
Examiner B & 2 & 9 & 11 \\
Examiner C & 6 & 27 & 33 \\
Total & & & \\
\hline
\end{tabular}

between the observed (Po) and the proportion of agreement expected by chance. Rates vary between -1.0 (complete disagreement) and +1.0 (complete agreement); zero represents the agreement expected by chance.

\section{RESULTS}

Table 1 shows the results of the examination by the three examiners employing the visual, radiographic and microCT scan methods and the inter-examiner agreement for occlusal lesion detection calculated by Cohen's Kappa rates. Kappa rates were $0.515,0.583$ and 0.924 for the inter-observer agreement, respectively for visual, radiographic and microtomographic examinations (Table $1)$.
Table 2 presents the coincidence between visual examination and micro-CT scan examination, and between the radiographic examination and micro-CT scan examination for three examiners. So that visual inspection and examination by micro-CT scan could be compared, the coincidence for the presence or the absence of communication from the external to the lesion for both techniques was taken into account.

Figure $1 \mathrm{a}, \mathrm{b}$ and $\mathrm{c}$ shows a new sequence of analysed images of tooth $F$. In this case, the examiners disagreed on visual examination and agreed on the radiographic and micro-CT examinations. One examiner stated that the clinical examination indicated a sound surface and the radiographic image was described as radiolucency in dentine, to which all examiners agreed (Figure $1 \mathrm{a}$ and $1 \mathrm{~b}$, 

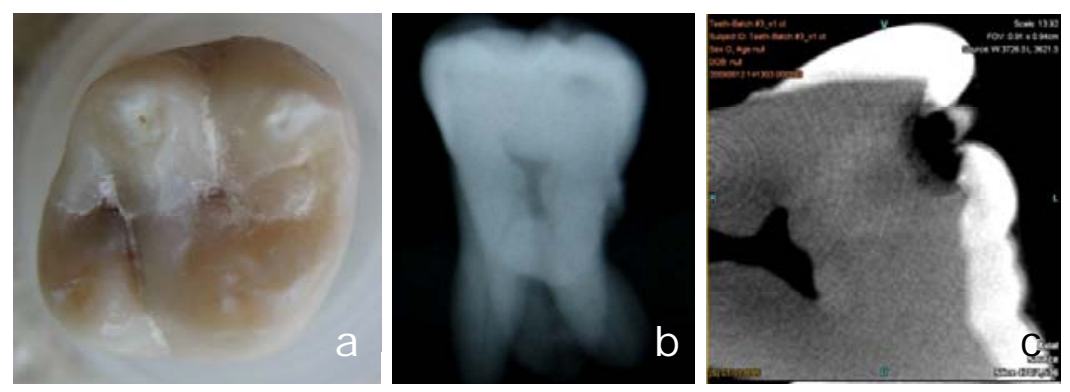

Figure 1. Tooth F: (a) photo of occlusal surface, (b) radiographic image, and (c) micro-CT scan image.
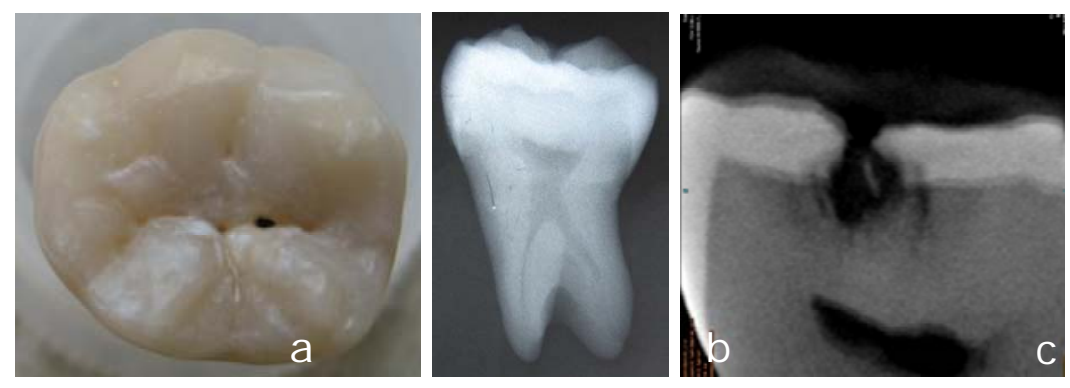

Figure 2. Tooth E: (a) photo of occlusal surface, (b) radiographic image, and (c) micro-CT scan image.
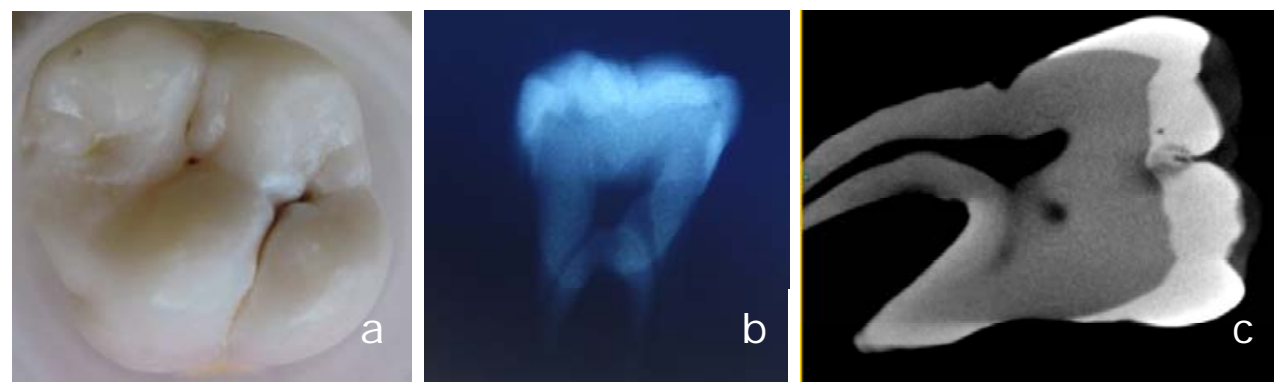

Figure 3. Tooth D: (a) photo of occlusal surface, (b) radiographic image, and (c) micro-CT scan image.

respectively). The micro-CT image revealed radiolucency in enamel and dentine, to which all the three examiners agreed (Figure 2c).

Tooth $E$ is as shown in Figure $2 a, b$, and $c$. The three examiners agreed that there was a lesion in the occlusal surface when examined visually (Figure 2a). However, they failed to agree on the radiographic diagnosis (Figure $2 b$ ). All examiners detected the presence of radiolucency in enamel and dentine with micro-CT scan (Figure 2c).

With regard to tooth $\mathrm{D}$, as shown in Figure $3 a, b$, and $c$, the examiners differed on the visual and radiographic examinations (Figure $3 a$ and b, respectively). However, when the micro-CT scan examination was employed, the three examiners were able to identify radiolucency in enamel and dentine.

\section{DISCUSSION}

In the present research, occlusal lesions were determined by three methods of caries detection that enabled dental practitioners to decide on treatment options, of which two are used routinely in dental practice whilst the third method, micro-CT scan image, was chosen as the gold standard owing to its superior quality and accuracy. Results indicated that the visual and radiographic method exhibited only moderate correlation to micro-CT scan (Tables 1 and 2). The images observed by micro-CT scan revealed that the 11 teeth examined in current research showed communication between the external environment and the occlusal lesion. Communication with the external environment detected 
by micro-CT scan invalidated the theory that described these injuries as hidden caries (Seow, 2000; Chong et al., 2003), since, by definition, this condition would definitely show dentine lesion under non-cavitated enamel. Interestingly enough, micro-CT scan showed broken enamel with dentine exposed to mouth environment. The low sensitivity and specificity of the conventional radiographs for the detection of lesions in the occlusal surface may be due to the nature of the twodimensional, albeit low-quality, images (Poorterman et al., 2000).

When caries reached the dentin at the dentin-enamel junction, decay quickly spread laterally. Decay within the dentin followed a triangular pattern that pointed towards the tooth's pulp. In fact, decay pattern is typically described as two triangles (one triangle in enamel and the other in dentin) with their bases joined to each other at the dentin-enamel junction (DEJ). Actually, such baseto-base pattern is typical of pit and fissure caries (Thylstrup and Fejerkov, 1994). It should be emphasized that the radiograph-generated image failed to provide a clear view of this characteristic of occlusal caries, clearly seen on the micro-CT scan image.

Clinical diagnosis of occlusal caries is one of the most difficult issues in the field of dentistry, with uncertainties as to its outcome. Mouth mirror, good reflected light and a blunt or a sharp probe are the dentist's principal diagnostic tools, but they lack overall accuracy in terms of sensitivity and specificity (Poorterman et al., 2000). This difficulty has also been observed in current study on the diagnosis of the eleven examined teeth. For instance, when examiners diagnosed tooth $\mathrm{F}$ (Figure 1 ), they could only partially agree on the visual examination, whereas they totally agreed on the radiographic and micro-CT scan examination. In fact, Examiner 1 described the occlusal surface as sound, but Examiners 2 and 3 identified lesion on the same surface (Figure 1a). While the three examiners identified radiolucency in enamel and dentine by micro-CT scan (Figure 1c), the radiographic examination only allowed them to diagnose radiolucency in dentine (Figure $1 \mathrm{~b}$ ). Dental X-rays mostly fail on displaying enamel lesions and communication with the external environment. They were, however, very clear in the images produced by micro-CT scan (Table 2).

Although the three examiners were able to clinically identify lesion on the occlusal surface (Figure 2a), the dental radiographs were not helpful to show to Examiner 1 the radiolucency suggestive of lesion in dentine. Contrastingly, Examiners 2 and 3 identified radiolucency in dentine (Figure 2b) but micro-CT scan images failed to confirm any diagnosis by the radiographic examination (Table 2). In fact, it showed the presence of radiolucency in enamel and dentine (Figure 2c) and reflected the communication of the lesion with the external environment and thus confirmed only the findings of the visual diagnosis. Examination of tooth $\mathrm{E}$ confirmed the limitations of the dental radiographs previously mentioned and stressed by current research. The aforementioned also indicated the importance of accurate clinical examination (Zafersoy-Akarslan et al., 2009) confirmed by agreement between clinical and micro-CT scan examinations for this tooth.

Visual and radiographic examinations were not accurate enough for inter-examiner agreement in the case of some teeth (Table 1 ). The diagnosis of tooth $D$ is highly relevant to illustrate this fact (Figure $3 a, b$, and c). This observation reflected the difficulties of clinical examination and also showed the limitations of dental radiographs when compared with micro-CT scan images. In this context, the hidden caries lesions were not easily visualized by dental radiograph, because the visibility of caries was determined by the ratio of enamel to caries through which $\mathrm{x}$-rays penetrate. Radiographic diagnosis of caries must always be supplemented with a careful clinical examination (Wenzel, 2014).

Visual examination, routinely used for detecting caries in dental clinics, has also been used in other studies (Souza-Zaroni et al., 2006; Zafersoy-Akarslan et al., 2009). In current research, the occlusal surfaces were graded according to each examiner's clinical experience to demonstrate the lack of consensus diagnosis among the different dentists. This hypothesis became clear when the Kappa for inter-examiner rate $(K=0.515)$ was employed (Table 1). It actually established only moderated agreement and became a reflection of the difficulty mentioned in several studies to perform more accurate clinical caries diagnoses on occlusal surface (Barder, 2002). Further, the observers hailed from different training and routine, and thus with different degrees of experience in detecting caries. Low interobserver agreement could be related to these factors and variation in caries detection among dentists is a common phenomenon (Zafersoy-Akarslan et al., 2009). There is no guarantee that the examiners will agree on diagnoses, since their decisions are based on previously acquired knowledge and experience. Since examiners' experience obviously influences the diagnostic, it is important to highlight that the comparison of results involves subjective aspects, such as knowledge and clinical experience (Souza-Zaroni et al., 2006).

The reported specificity of visual examination for detecting occlusal lesion was described as high by various authors. Although in current research, all the teeth were considered sound by visual examination, CT scan images showed radiolucency without any doubt (Table 2). This fact also indicated that the various theories labelling this type of lesion as 'fluoride syndrome', 'fluoride bomb' and 'hidden caries' (McDonald and Sheiham, 1992; BeltránAguilar et al., 1999; Whelton, 2004; Bernabé, 2009; Bagramian et al., 2009) may be nothing more than a misdiagnosis for hidden occlusal caries merely relying on visual and dental radiographic examination. Moderate inter-examiner agreement in current investigation occurred for radiographic examination $(\mathrm{K}=0.583)$ (Table 1$)$. 
Since the examinations were performed by the aforementioned technique, the diagnoses with an identified lesion only on dentin were predominant (Tables 1). Consequently, the lesion which extended from the enamel, as displayed by micro-computed tomography examination, was not perceived.

On the other hand, micro-CT scans identified lesions in dentin and enamel on occlusal surfaces more clearly than the traditional radiographic survey. This might be perceived when Kappa inter-examiners rates (K=0.924) were provided (Table 1). However, our findings are not in support of the routinely recommendation for the use of micro-CT scan in daily clinical use due to the large amount of ionizing radiation emitted by this examination. This in vitro study aimed to verify the limitations of conventional tests for the detection of hidden caries.

\section{Conclusion}

Results of this work indicate that there is strong limitation for accurate diagnosis of hidden caries due to conventional examinations limitations, visual and dental radiography. New imaging and other more advanced technologies are needed to improve those procedures.

\section{Conflict of interests}

Authors declare that they have no conflict of interest, nor any interference with the results of this article.

\section{ACKNOWLEDGEMENTS}

The authors would like to express their gratitude to the teachers, school staff, and parents who participated in the survey. This study was supported by a grant from the Brazilian Ministry of Education (CAPES).

\section{REFERENCES}

Bader JD, Shugars DA, Bonito AJ (2002). A systematic review of the performance of methods for identifying carious lesions. J. Public Health Dent. 62(4):201-213.

Bagramian RA, Garcia-Godoy F, Volpe AR (2009). The global increase in dental caries. A pending public health crisis. Am. J. Dent. 22:3-8.

Beltrán-Aguilar ED, Estupiñán-Day S, Báez R (1999). Analysis of prevalence and trends of dental caries in the Americas between the 1970s and 1990s. Int. Dent. J. 49(6):322-329.
Bernabé E, Sheiham A, Sabbah W (2009). Income, income inequality, dental caries and dental care levels: an ecological study in rich countries. Caries Res. 43(4):294-301.

Chong MJ, Seow WK, Purdie DM, Cheng E, Wan V (2003). Visualtactile examination compared with conventional radiography, digital radiography, and Diagnodent in the diagnosis of occlusal occult caries in extracted premolars. Pediatr. Dent. 25(4):441-449.

Creanor SL, Russell JI, Strang DM, Stephen KW, Burchell CK (1990). The prevalence of clinically undetected occlusal dentine caries in Scottish adolescents. Br. Dent J. 169(5):126-129.

Hashizume LN, Mathias TC, Cibils DM, Maltz M (2013). Effect of the widespread use of fluorides on the occurrence of hidden caries in children. Int. J. Paediatr. Dent. 23(1):72-76.

McDonald SP, Sheiham A (1992). The distribution of caries on different tooth surfaces at varying levels of caries - a compilation of data from 18 previous studies. Community Dent. Health. 9(1):39-48.

Mutsvari T, García-Zattera MJ, Declerck D, Lesaffre E (2012). Dealing with misclassification and missing data when estimating prevalence and incidence of caries experience. Community Dent. Oral Epidemiol. 40(1):28-35.

Neuhaus KW, Ellwood R, Lussi A, Pitts NB (2009). Traditional lesion detection aids. Monogr. Oral Sci. 21:42-51.

Parks ET (2000). Computed tomography applications for dentistry. Dent. Clin. North Am. 44(2):371-394.

Poorterman JH, Weerheijm KL, Groen HJ, Kalsbeek H (2000). Clinical and radiographic judgement of occlusal caries in adolescents. Eur. J. Oral Sci. 108(2):93-98.

Ricketts DN, Ekstrand KR, Martignon S, Ellwood R, Alatsaris M, Nugent $Z$ (2007). Accuracy and reproducibility of conventional radiographic assessment and subtraction radiography in detecting demineralization in occlusal surfaces. Caries Res. 41(2):121-128.

Seow WK (2000). Pre-eruptive intracoronal resorption as an entity of occult caries. Pediatr. Dent. 22(5):370-376.

Souza-Zaroni WC, Ciccone JC, Souza-Gabriel AE, Ramos RP, Corona SA, Palma-Dibb RG (2006). Validity and reproducibility of different combinations of methods for occlusal caries detection: an in vitro comparison. Caries Res. 40(3):194-201.

Thylstrup A, Fejerkov O (1994). Radiologic diagnosis in caries management. Copenhagen, Munsgaard. pp. 367-382.

Weerheijm KL (1997). Occlusal 'hidden caries'. Dent. Update. 24(5):182-184.

Weerheijm KL, Gruythuysen RJ, van Amerongen WE (1992). Prevalence of hidden caries. ASDC J. Dent. Child. 59(6):408-412.

Wenzel A (2014). Radiographic display of carious lesions and cavitation in approximal surfaces: Advantages and drawbacks of conventional and advanced modalities. Acta Odontol. Scand. 72(4):251-264.

Whelton $\mathrm{H}$ (2004). Overview of the impact of changing global patterns of dental caries experience on caries clinical trials. J Dent Res. 83 Spec No C:C29-34.

Zadik Y, Bechor R (2008). Hidden occlusal caries: challenge for the dentist. N. Y. State Dent J. 74(4):46-50.

Zafersoy-Akarslan Z, Erten H, Uzun O, Semiz M (2009). Reproducibility and agreement of clinical diagnosis of occlusal caries using unaided visual examination and operating microscope. J. Can. Dent. Assoc. 75(6):455. 